\title{
Can Poor Oral Health Cost You High Blood Sugar?
}

\section{Bharat Joshi*}

Assistant Professor and Editor, Department of Periodontology, Swami Devi Dyal Hospital and Dental College, Haryana, India

*Corresponding Author: Assistant Professor and Editor, Department of Periodontology, Swami Devi Dyal Hospital and Dental College, Haryana, India

Received: October 14, 2019; Published: November 04, 2019

DOI: $10.31080 /$ ASDS.2019.03.0691

A significant question arises in the mind of individuals that is oral health really important for maintenance of overall systemic health. Well I can say yes because many studies have supported the relationship of gum diseases (periodontitis) with systemic conditions like high blood sugar (diabetes) and blood pressure (Hypertension).This is really interesting to appreciate that there is two way relationship between the two conditions which means one is responsible for other. It is found that Diabetes (especially uncontrolled) predisposes the oral cavity to excess bone destruction (both vertical and horizontal bone loss) and pus formation (Periodontal Abscess) thereby making an entrance for gram negative bacteria (Porphyromonas Gingivalis and Fusobacterium Nucleatum) to invade. Reason behind is that high blood sugar decreases the basal metabolic rate which in turn decreases the body immunity. Decrease in immunity alters the host response and make entry of pathogenic bacteria (Porphyromonas Gingivalis and Fusobacterium Nucleatum) comparatively very easy. Similarly presence of periodontitis also paves the way for systemic conditions like diabetes because the endotoxins released by these bacteria cause inflammation and consequently, there is release of inflammatory mediators (PGE2 and TNF- $\alpha$ ).These mediators cause excessive bone destruction and increases chances of insulin resistance. We all know that Diabetes already has 5 complications like retinopathy, neuropathy and nephropathy but now days periodontitis is considered as the $6^{\text {th }}$ complication of diabetes. Both are strongly linked with each other and therefore, in my opinion, proper oral health is must for systemic conditions. Any imbalance between the two can affect the individual in a very bad manner. Hence, every effort should be done to maintain good oral hygiene like proper brushing, recall after every 3 - 6 months and selective but balanced diet. In this way we can prevent oral diseases and maintain our systemic health.

\section{Volume 3 Issue 12 December 2019 (C) All rights are reserved by Bharat Joshi.}

